\title{
Vorwort zur 13. Auflage
}

Der bisherige Herausgeber, Landgerichtsrat Dr. Walter Petters, verstarb am 18. 1. 1963, kurz vor Vollendung seines 75. Lebensjahres. Nachdem ich bereits an der 1961 erschienenen 7. Auflage der „Strafprozeßfälle" und an der 1962 erschienenen Vorauflage der "Strafrechtsfälle" mitgearbeitet hatte, war es sein Wunsch, daß ich sein Werk fortführen möge. Diesem Wunsch bin ich gerne gefolgt. 1965 erschien die 25. Auflage des "Strafgesetzbuchs" (Lehrkommentar mit Erläuterungen und Beispielen), 1966 folgte die 8. Auflage der „Strafprozeßfälle“. Mit der jetzt vorliegenden Neuauflage der "Strafrechtsfälle" ist das Gesamtwerk wieder auf den neuesten Stand gebracht.

Wie bei der Neubearbeitung der beiden anderen Bände habe ich mich auch bei den "Strafrechtsfällen" von dem Bestreben leiten lassen, Charakter und System des vor allem in Referendarkreisen so beliebten Buchs, von dem schon Professor Ebermayer vor mehr als drei Jahrzehnten sagte, es sei für jeden jungen Juristen fast unentbehrlich, so weit wie möglich zu erhalten. Trotzdem ließ es sich nicht umgehen, das Buch völlig neu zu gestalten. Die seit der 10. Auflage vorgenommene Aufteilung des Werkes in zwei Teile mit insgesamt 24 Fällen hatte sich auf die Dauer doch nicht bewährt. Einem vielfach geäußerten Leserwunsch folgend habe ich deshalb den Umfang wieder auf einen Band mit nur 16 Fällen beschränkt und dabei nach dem Grundsatz "multum, non multa" auf die Erörterung weniger wichtiger Problemkreise verzichtet. Diese Beschränkung bot Anlaß und Gelegenheit, völlig neue Fälle zu bilden.

Während die Fälle der Vorauflage noch auf Entscheidungen des früheren Reichsgerichts aufgebaut waren, sind die neuen Fälle ausschließlich an der Rechtsprechung des Bundesgerichtshofs ausgerichtet, die bis Ende 1967 (Heft 5 des 21. Bands der amtlichen Sammlung) verarbeitet wurde. Die Umstellung auf die Judikatur des Bundesgerichtshofs stellte nicht nur neue Rechtsprobleme in den Vordergrund, sondern bot gleichzeitig auch die Möglichkeit einer zeitgemäßen und lebensnahen Fallgestaltung. Bei der Neufassung der Fälle und ihrer Lösungen kam mir meine langjährige Erfahrung in der Ausbildung des juristischen Nachwuchses zugute. 
Die Lösungen mit ihren Vorbemerkungen gehen wie bisher weit über den einzelnen Fall hinaus, um einen möglichst umfassenden Überblick über die jeweils angeschnittenen Problemkreise zu verschaffen. Sie sind vor allem an der höchstrichterlichen Rechtsprechung ausgerichtet. Aber auch das Schrifttum wurde, soweit erforderlich, mehr als bisher beriücksichtigt.

$\mathrm{Zu}$ den wesentlichen Neuerungen gehört die strengere Trennung der eigentlichen Lösungen von den der jeweiligen Lösung vorangestellten Vorbemerkungen. Die Lösungen werden dadurch übersichtlicher und einprägsamer, ohne daß die bisherige Methodik der Fallsammlung als induktives Lehrbuch entscheidend berührt wird. In der jetzt gewählten Form stellt sich das Buch als Kombination von Lehrbuch, Kommentar und Fallsammlung dar. Im Vordergrund steht, wie bisher, der Fall. Gleichzeitig aber werden alle wesentlichen Probleme des Allgemeinen Teils lehrbuchmäßig den Lösungen vorangestellt (S. 1 bis 71). Die für die Prüfung wichtigen Schwerpunkte des Besonderen Teils werden kommentarmäßig in den Vorbemerkungen der Fälle abgehandelt, in denen sie von rechtlicher Bedeutung sind.

Alle Anderungen erfolgten in dem Bestreben, dem Buch das eingangs erwähnte Prädikat von Ebermayer zu erhalten und dem Studierenden bzw. Referendar den für die Prüfung im Strafrecht erforderlichen Stoff in leicht verständlicher und einprägsamer Form näher zu bringen und ihn mit allen wesentlichen Problemen vertraut zu machen.

Pforzheim, im Dezember 1967

Holger Preisendanz 